\title{
REGISTROS NOTABLES DE MAMÍFEROS PARA LA RESERVA DE LA BIÓSFERA SIERRA GORDA DE GUANAJUATO, MÉXICO
}

\author{
Oscar Sosa-Guerrero ${ }^{1}$, José I. Campos-Rodríguez ${ }^{1}$, Xhail Flores-Leyva", \\ Paola Yáñez-López ${ }^{1}$ y Leticia A. Mora-Villa ${ }^{2}$
}

${ }^{1}$ Laboratorio de Cordados Terrestres, Departamento de Zoología, Escuela Nacional de Ciencias Biológicas, Instituto Politécnico Nacional, Prolongación de Carpio y Plan de Ayala s/n, Ciudad de México, México.

${ }^{2}$ Laboratorio de Ecología Vegetal, Departamento de Botánica, Escuela Nacional de Ciencias Biológicas, Instituto Politécnico Nacional. Prolongación de Carpio y Plan de Ayala s/n, Ciudad de México, México.

Autor de correspondencia: José I. Campos-Rodríguez, jicamposrodriguez@ hotmail.com

\section{ABSTRACT}

We present distribution range extentions for five species of mammals from the Sierra Gorda de Guanajuato Biosphere Reserve in state of Guanajuato, Mexico. These are two felids: Lynx rufus and Puma concolor, as well as the western speckled skunk Spilogale gracilis, the peccari Dicotyles crassus, and the coati Nasua narica. The presence of these records reflects the importance of the Sierra Gorda de Guanajuato Biosphere Reserve as one of the main protected natural areas of Guanajuato, Mexico, since this area constitutes a natural bridge between species of neartic and neotropical affinity. This type of study reaffirms the need to continue conducting regional and local biological inventories in Mexico.

Key words: Range extensión, camera-traps, Victoria, Guanajuato, biological corridor.

Palabras clave: Distribución, cámaras trampa, Victoria, Guanajuato, corredores biológicos. 
Guanajuato se caracteriza por su orografía heterogénea y su ubicación en la zona de transición entre la región Neártica y la Neotropical, lo cual ha favorecido la presencia de una mastofauna compleja, algunos de cuyos elementos alcanzan su límite de distribución en esta región. Debido a su variada composición mastofaunística, esta entidad ha sido objeto de valiosas aportaciones en los últimos años. A la fecha, se han reportado 93 especies de mamíferos pertenecientes a 20 familias (Sánchez et al., 2014; 2016), cifra que ubica a la entidad como de riqueza intermedia para el país, según Sánchez et al., (2012). Una de las zonas con mayor diversidad de mamíferos en la entidad es la Reserva de la Biósfera Sierra Gorda de Guanajuato (RBSGG). Esta área natural protegida fue decretada en 2004, se localiza en el noreste del estado y comprende los municipios de San Luis de la Paz, Victoria, Xichú y Santa Catarina (semarnat, 2007). Las contribuciones más recientes sobre la mastofauna de la RBSGG son las de Iglesias-Hernández et al., (2008), Sánchez et al., (2009), Elizalde-Arellano et al., (2010), Magaña-Cota et al., (2010), Charre-Medellín et al., (2012a; 2012b; 2016), Cecaira-Ricoy et al., (2012), Iglesias-Hernández et al., (2012) y Mora-Villa et al., (2014). Dichos trabajos se han enfocado en el estudio de mamíferos medianos y grandes mediante el empleo de cámaras-trampa, en los municipios de Victoria y Xichú (Iglesias-Hernández et al., 2012; Charre-Medellín et al., 2012b; Charre-Medellín et al., 2016); en el estudio de la diversidad de comunidad de murciélagos en San Luis de la Paz (Mora-Villa et al., 2014), así como en la publicación de nuevos registros o extensiones de distribución de varias especies de mamíferos para Guanajuato (Iglesias-Hernández et al., 2008; Sánchez et al., 2009; Elizalde-Arellano et al., 2010; Magaña-Cota et al., 2010; Cecaira-Ricoy et al., 2012; Charre-Medellín et al., 2012a). El objetivo del presente trabajo es dar a conocer cinco extensiones notables de distribución de mamíferos, todas ellos procedentes de la RBSGG.

El área de estudio es la cañada del arroyo El Rejalgar y la parte alta del río Manzanares, en las inmediaciones de los poblados de Mesita del Tigre y la Mora, municipio de Victoria Guanajuato, dentro de la zona de amortiguamiento de la RBSGG, en el noreste del estado (Figura. 1). El intervalo altitudinal va de 1,660 msnm en el fondo de la cañada a 1,900 $\mathrm{msnm}$ en las partes más altas. La vegetación está conformada por un complejo mosaico de pastizales naturales en las partes altas; chaparral y bosque de encino en laderas y escurrimientos, así como bosque de galería y matorral submontano en las partes bajas de la cañada (CONANP, 2005; SEMARNAT, 2007).

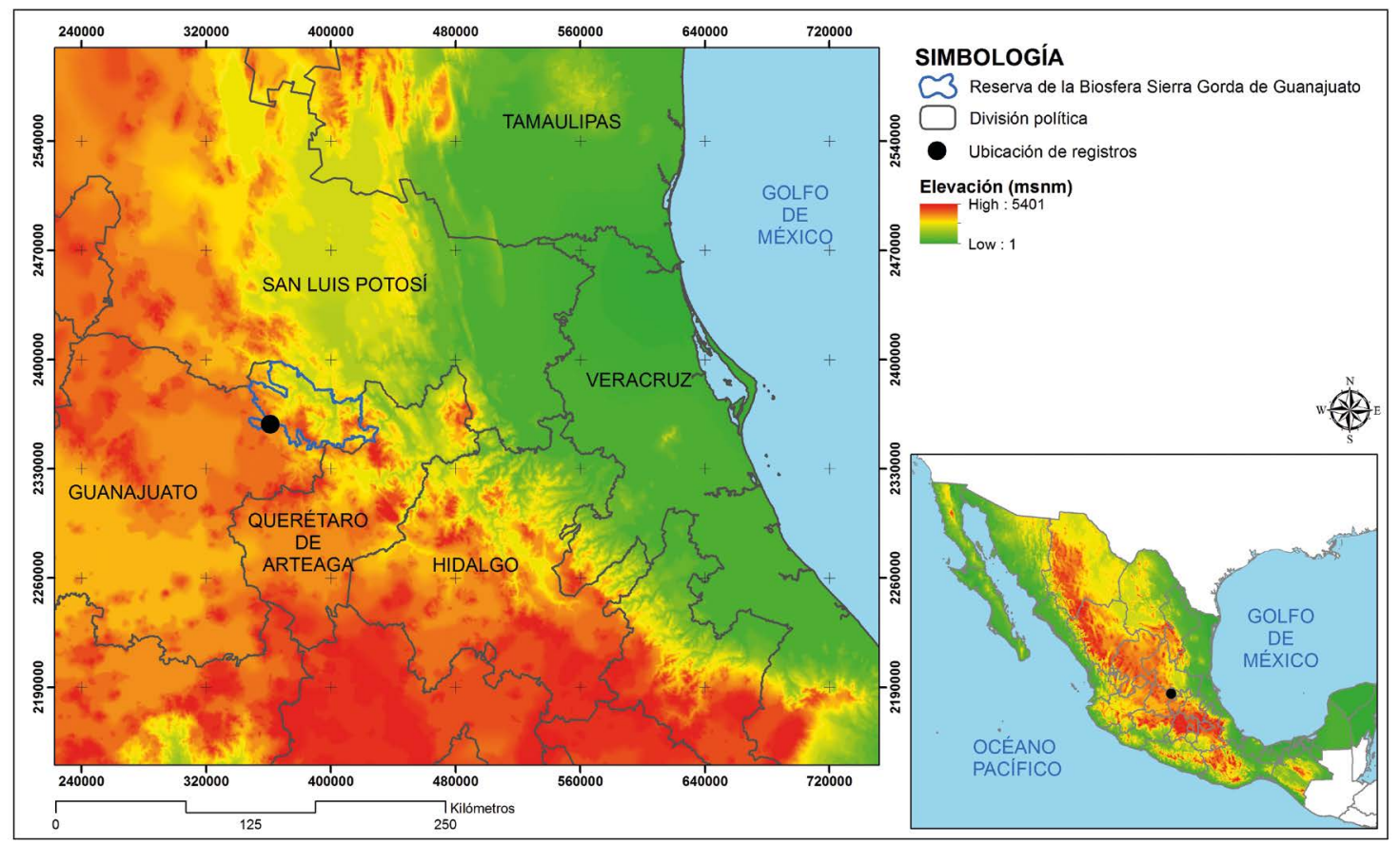

Figura 1. Localizacion del area de estudio en el estado de Guanajuato, indicada por el punto negro. 
El arroyo El Rejalgar es un tributario del río Manzanares, que a su vez aporta su afluente al río Santa María en la cuenta hidrológica Río Moctezuma. El clima es BS1k" semiárido, templado, con temperatura media anual entre $12^{\circ} \mathrm{C}$ y $18^{\circ} \mathrm{C}$. La temperatura del mes más frío oscila entre $-3^{\circ} \mathrm{C}$ y $18^{\circ} \mathrm{C}$ y precipitación anual menor a $400 \mathrm{~m}$ (CONANP, 2005). Como parte de un estudio sistemático dirigido a obtener un inventario de mamíferos medianos y grandes en la cañada del arroyo El Rejalgar, situado entre las comunidades de La Mora y Mesita del Tigre, (Figura 1) municipio de Victoria, Guanajuato. Se colocaron 12 fototrampas: dos de la marca Moultre® A-5 Trail Camera $\left(\operatorname{Gen}_{2}\right)$, dos Wildgame InnovationsTM Buck Commander Nano 8, dos Cuddeback® modelo Long Range IR Model E2 y seis ScoutGuard SG570-12mHD, las cuales fueron dispuestas en 24 estaciones de muestreo. Estas fueron colocadas a una altura no mayor de $40 \mathrm{~cm}$ del nivel del suelo (Chávez et al., 2013). Las cámaras fueron programadas para captar fotografías con tres series de fotos por evento y permanecer activas durante 24 horas. La posición de cada una fue georreferenciada con un geoposicionador modelo Garmin ® GPSMAP 64s. Ocho fototrampas contaban con sensores infrarrojos y cuatro con flash. Las fototrampas fueron dispuestas en sitios donde previamente fueron detectados senderos de mamíferos medianos, que a su vez fueron reconocidos por la presencia de huellas, excretas o echaderos, así como con ayuda de guías locales. Las cámaras trampas fueron revisadas una vez cada dos meses, en cada fotografía obtenida se imprimió la hora y la fecha. Se obtuvo el esfuerzo total de muestreo multiplicando el número total de estaciones por el total de días de muestreo (Medellín et al., 2006). Se efectúo una revisión de los registros de mamíferos procedentes de la RBSGG Guanajuato contenidos en el portal de Foto Colectas Biológicas (CFG: FB) del Instituto de Biología de la UNAM (www.unibio.unam.mx), la plataforma Naturalista (www.naturalista.mx), la red Global Biodiversity Information Facility (www.gbif.org) y la literatura especializada. Se siguió a Ramírez-Pulido et al., (2014) para cuestiones de nomenclatura. Se reportan cinco nuevos registros de mamíferos medianos a grandes para la RBSGG, los cuales son: Lynx rufus (Schreber, 1777), Puma concolor (Linnaeus, 1771), Spilogale gracilis Merriam 1890, Nasua narica (Linnaeus, 1776) y Dicotyles crassus (Merriam, 1901). Los cuales fueron obtenidos con un esfuerzo total de muestro de 218 días/trampa entre los meses de julio de 2016 a febrero de 2017 y un esfuerzo mensual de 737.14 horas/ mes. Se obtuvieron 1,502 fotografías de mamíferos medianos a grandes.

\section{Lynx rufus (Carnivora: Felidae)}

Se obtuvieron 18 fotografías de gato montés correspondientes a ocho individuos. Los registros proceden de las siguientes coordenadas geográficas: $21^{\circ} 18$ '52.5" latitud N y $-100^{\circ} 20^{\prime} 10.1^{\prime \prime}$ longitud W, el 30 de septiembre de 2016, 11 de noviembre de 2016 y 13 de enero de $2017 ;-100^{\circ} 20^{\prime 20.1 "}$ "

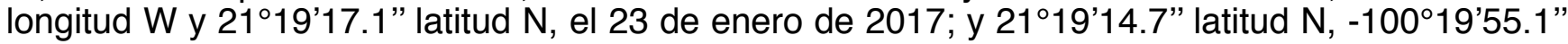
longitud W, el 27 de octubre de 2016. La especie fue registrada en matorral crasicaule, bosque de encino y chaparral, en un intervalo altitudinal de 1,720 a 1,947 msnm. En la RBSG ha sido registrada previamente en tres localidades del municipio de Victoria: Cañuelas, Los Colorados, EI Sabino, situadas aproximadamente a 29.2 y 32.5 km al NE de La Mora (Charre-Medellín et al., 2012b).

\section{Puma concolor (Carnivora: Felidae)}

Se fotografío un ejemplar procedente del sitio con coordenadas $21^{\circ} 18^{\prime} 52.5^{\prime \prime}$ latitud $\mathrm{N} \mathrm{y}-100^{\circ} 19^{\prime}$ 28.4" longitud W, el 20 de diciembre de 2016. El registro se obtuvo en bosque de encino, a una altitud de 1,957 msnm. En la RBSGG, existen registros de puma en las cercanías de El Platanar en el municipio de Xichú (Iglesias-Hernández et al., 2012) así como en las comunidades de San Isidro de las Palmas, El Sabino y Derramaderos en el municipio de Victoria (Charre-Medellín et al., 2012). Nuestro registro se localiza a $19.8 \mathrm{~km}$ al W del registro más cercano que es Derramaderos (Charre-Medellín et al., 2012b). El ejemplar fue observado en el límite oeste de la RBSGG.

\section{Spilogale gracilis (Carnivora: Memphitidae)}

Fue encontrado en bosque de galería con una fotografía correspondiente a un solo ejemplar el 25 de octubre de 2016 en $21^{\circ} 19^{\prime} 0.7 .5^{\prime \prime}$ latitud N y $100^{\circ} 19^{\prime} 55.8^{\prime \prime}$ longitud W, a 1,790 msnm. Se trata del segundo registro para la RBSGG, la presencia de la especie en la entidad ha sido ampliamente 


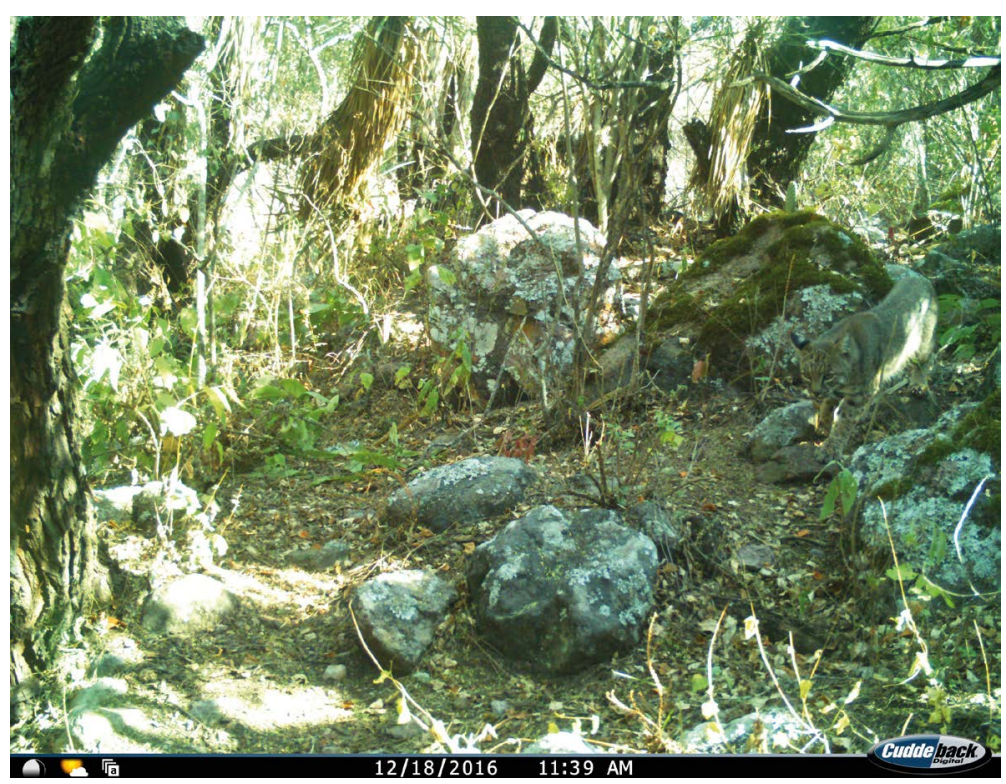

Figura 2. Gato montés (Lynx rufus).

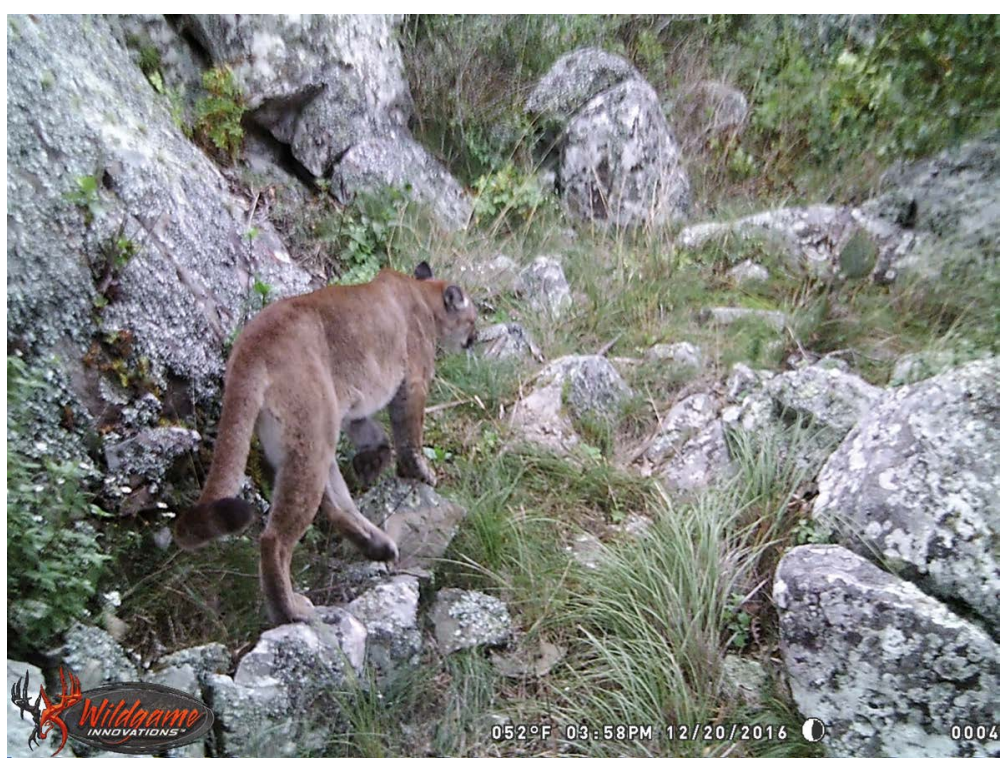

Figura 3. Puma (Puma concolor).

documentada por Sánchez et al., (2014). El registro más cercano se ubica a $32.9 \mathrm{~km}$ al NNE, en la comunidad de El Roblar (Cecaira-Ricoy et al., 2012).

\section{Nasua narica (Carnivora: Procyonidae)}

Se detectó en una estación de muestreo con coordenadas $21^{\circ} 20^{\prime} 06.0$ " latitud N y $101^{\circ} 19^{\prime} 08.9^{\prime \prime}$ longitud W, el 22 de septiembre de 2016 con seis fotografías de siete organismos distintos. Fue registrada en bosque de galería a 1,667 $\mathrm{msnm}$. La especie ha sido registrada en seis localidades dentro de la RBSGG, en los municipios de Victoria y Xichú en las comunidades de Victoria, El Platanal y Xichú (Charre-Medellín et al., 2012b; Iglesias-Hernández et al., 2012). El registro más cercano se ubica a $26.09 \mathrm{~km}$ al NNE, de la localidad de Cañuelas (Charre-Medellín et al., 2012b). 


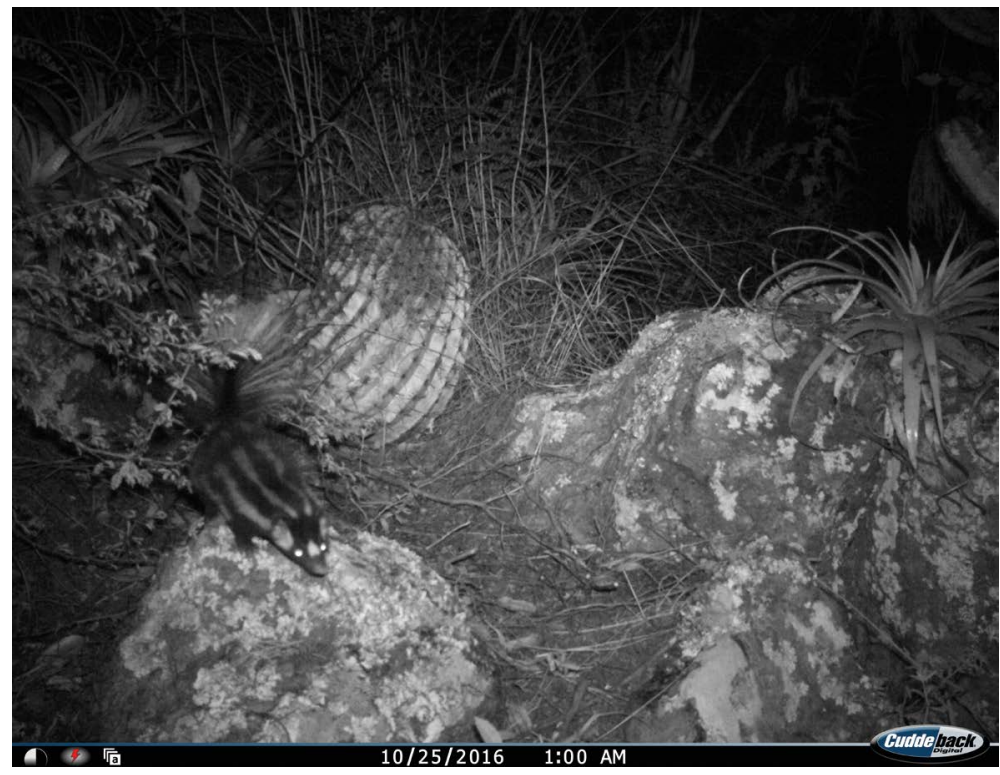

Figura 4. Zorrillo manchado (Spilogale gracilis).

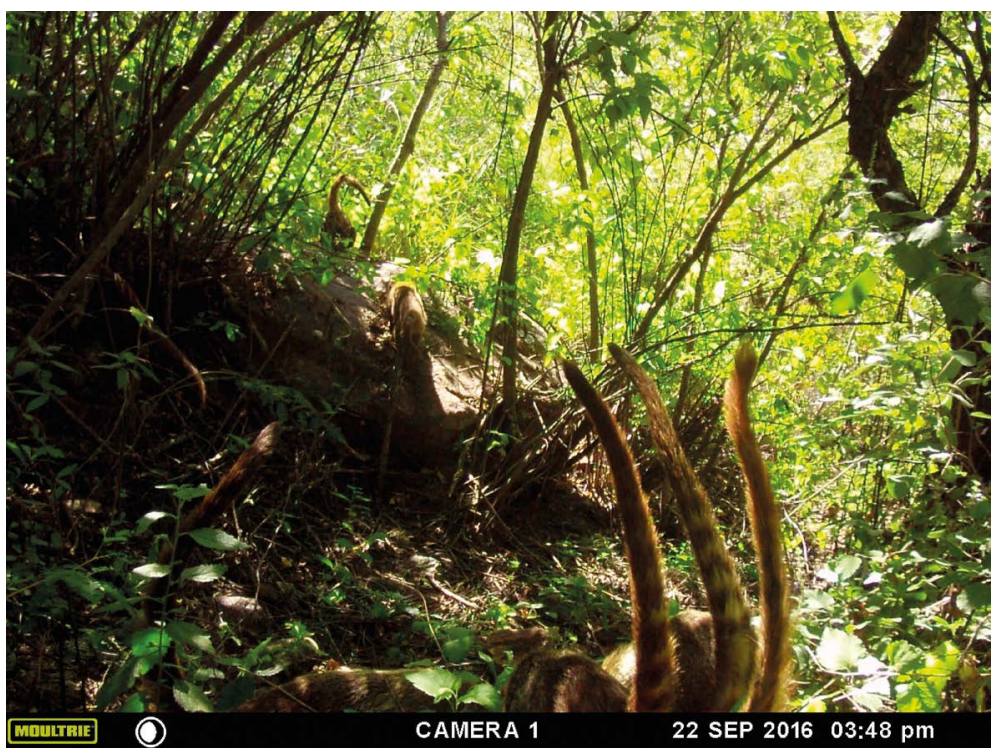

Figura 5. Tropa de coaties (Nasua narica).

\section{Dicotyles crassus (Artiodactyla: Tayassuidae)}

Se obtuvieron dos fotografías de pecarí (D. crassus) en dos estaciones de muestreo situadas en bosque de galería y fue posible identificar a dos individuos respectivamente en las siguientes coordenadas geográficas: $-100^{\circ} 18^{\prime} 55.1^{\prime \prime}$ longitud W, 21 ${ }^{\circ} 10^{\prime} 17.1^{\prime \prime}$ latitud N, el 27 de noviembre de 2016 y $-21^{\circ}$ 19'11.3" latitud Norte y $-100^{\circ} 19^{\prime} 55.4^{\prime \prime}$ longitud W el 28 de noviembre de 2016, entre 1,720 a 1,762 msnm. Éste es el segundo registro para el municipio de Victoria. Ésta especie había sido registrado previamente por Charre-Medellín et al., (2012b), también ha sido registrada al norte de Xichú (www. naturalista.mx), en ambos casos sin proporcionar una localidad específica de colecta. Este registro se sitúa en los límites de la provincia biótica de la Sierra Madre Oriental y el Altiplano Mexicano, en el extremo oeste de la RBSGG, siendo el registro más occidental reportado para la especie. 


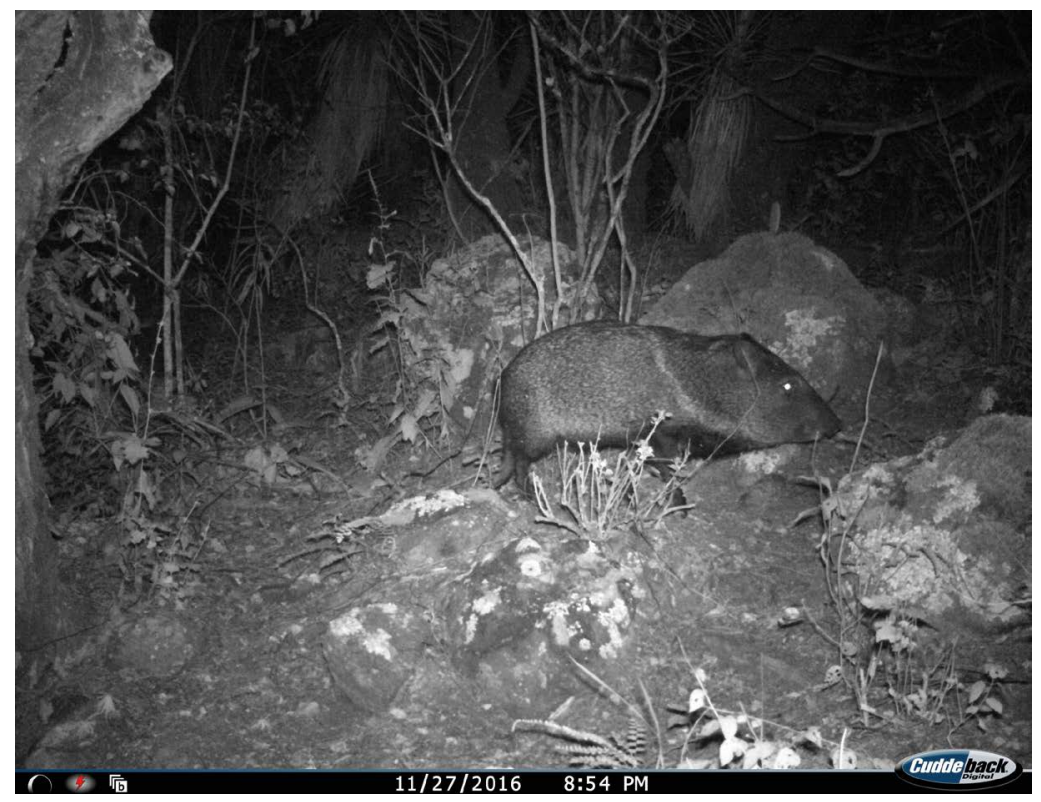

Figura 6. Pecarí (Dicotyles crassus).

Los estudios de fototrampeo de mamíferos medianos y grandes en la RBSGG se han concentrado principalmente en la zona núcleo, en serranías agrestes y de difícil acceso situadas en los municipios de Victoria y Xichú. Nuestro estudio es el primero enfocado a la zona de amortiguamiento de la RBSGG, en los límites de las provincias bióticas Sierra Madre Oriental y Altiplano Mexicano. El arroyo El Rejalgar es uno de los principales afluentes del río Manzanares, que su vez es tributario del río Santa María, dentro de la cuenca del río Moctezuma. Los registros de $N$. narica y D. crassus son relevantes por tratarse de especies de mamíferos de afinidad tropical o subtropical, para las cuales el arroyo, El Rejalgar constituye la localidad más occidental en dirección este-oeste de la planicie costera del Golfo de México hacia el Altiplano Mexicano: la primera especie se encuentra muy bien adaptada a los bosques templados de las laderas de la vertiente oriental de Sierra Madre Oriental, en tanto que la segunda se ha adaptado a las zonas áridas del Neártico, existiendo numerosos registros en el sureste de Texas, Coahuila y Nuevo León, pero escasos registros en la Mesa Central (www.gbif.org). Su presencia en el arroyo El Rejalgar es indicativo de que han sido capaces de cruzar la Sierra Madre Oriental a través de los tributarios del río Santa María y otros tributarios del Moctezuma para alcanzar la zona semidesértica del Altiplano Mexicano. La Sierra Madre Oriental separa a las tierras bajas de la planicie costera del Golfo de México de las tierras altas del Altiplano Mexicano, constituyendo una barrera geográfica y climática. Un patrón semejante ha sido sugerido por Campos-Rodríguez et al., (2009a; 2009b; 2009c) quien refiere la presencia de especies de anfibios y reptiles de afinidad tropical a subtropical en el rio Manzanares y Ramírez-Albores et al., (2015) quienes señalan la presencia de una zona de contacto de aves de afinidad tropical distribuidas en el este de México con las de ambientes secos del Altiplano Mexicano en la RBSGG.

Dos de las especies registradas en este estudio: Spilogale gracilis y Lynx rufus se encuentran ampliamente distribuidas en la zona Neártica de México. Ambas especies son simpátricas con $N$. narica y $D$. crassus en el arroyo El Rejalgar, lo que permite suponer que dicho arroyo forma parte de una notoria zona de contacto entre los mamíferos de mediano a gran tamaño con afinidad tropical de la planicie costera del Golfo de México y Sierra Madre Oriental con las especies de ambientes xerófilos del Altiplano, dicha zona de contacto se localiza en el río Manzanares puesto que sobre su cauce se han reportado mamíferos de afinidad tropical y xerófila en simpatría (Iglesias-Hernández et al., 2008; Cecaira-Ricoy et al., 2012; Charre-Medellín et al., 2012b; Magaña-Cota et al., 2010; Elizalde-Arellano et al., 2010; Mora-Villa et al., 2014). 
Los registros de $P$. concolor y $L$. rufus son notorios, puesto que se trata de depredadores tope cuya presencia es indicativa de hábitats excepcionalmente conservados. En el arroyo El Rejalgar se ha registrado la presencia de águila real (Aquila chrysaetos) (Campos-Rodríguez et al., 2016) que confirma que en las partes altas del rio Manzanares existe poblaciones de depredadores tope asociados a remanentes bien conservados de bosque de táscate, bosque de encino y matorral submontano que ameritan ser estudiados a detalle. Esta zona ha sido propuesta como parte de la zona de amortiguamiento de la RBSGG en la elaboración del plan de manejo correspondiente, con una política de uso tradicional (CONANP, 2013), no obstante, es posible que sea necesario reconsiderar su categoría debido precisamente a la presencia de depredadores tope. Se advierten como principales amenazas: la cacería de subsistencia que es realizada por pobladores locales, la presencia de ganado que conlleva o conllevará en un futuro conflictos derivados de una posible depredación de ganado por puma, debido a que dicha especie fue registrada muy cerca de las comunidades de Mesita del Tigre y La Mora así como la probable existencia de conflictos derivados de la presencia de concesiones mineras en la mencionada zona de amortiguamiento de la RBSGG.

Los registros aportados en el presente estudio contribuyen a incrementar la riqueza específica de mamíferos medianos a grandes en el área siendo también una contribución más en la elaboración de un inventario mastozoológico sistemático a mediano plazo dentro de la RBSGG.

\section{AGRADECIMIENTOS}

A Constanza Danaee Jímenez-Guevara, Pilar Luevano Goméz y Diana Pamela García Martínez por su apoyo en campo, a la familia Sosa-Guerrero y los habitantes de la comunidad de Mesita del Tigre, Victoria, Guanajuato por las facilidades brindadas durante nuestra estancia en campo. A la Comisión Nacional de Áreas Naturales Protegidas (conANP). A dos revisores anónimos cuyos comentarios y sugerencias contribuyeron a mejorar el contenido de la presente contribución.

\section{LITERATURA CITADA}

Campos-Rodríguez J.I., C. Elizalde-Arellano, J.C. López-Vidal, G.F. Aguilar-Martínez, S.N. Ramos-Reyes y R. Hernández-Arciga. 2009a. Nuevos registros de anfibios y reptiles para Guanajuato, procedentes de la Reserva de la Biosfera "Sierra Gorda de Guanajuato" y zonas adyacentes. Acta Zoológica Mexicana (n. s), 25:269-282.

Campos-Rodríguez J.I., B. Pérez-Valera, L.E. Aguilar, C. Elizalde-Arellano, J.C. López-Vidal y R. Hernández-Arciga. 2009b. Registros notables de reptiles para Guanajuato, México. $R e-$ vista Mexicana de Biodiversidad, 81:203-204.

Campos-Rodríguez J.I., B. Pérez-Valera, L.E. Aguilar y R. Hernández-Arciga. 2009c. Extensiones de distribución de reptiles para el estado de Guanajuato. Vertebrata Mexicana, 22:13-16.

Campos-Rodríguez J.I., O. Sosa-Guerrero y X. Flores-Leyva. 2016. Avistamientos recientes del águila real (Aquila chrysaetos) en la Reserva de la Biósfera Sierra Gorda de Guanajuato, México y sus consecuencias en el Plan de Manejo. Huitzil Revista Mexicana de Ornitología, 17:192-197.

Cecaira-Ricoy R., J.A. Iglesias-Hernández, J.F. Charre-Medellín, R. Bolaños-Martínez, G.E. Magaña-Cota, V. Sánchez-Cordero, E. Kato-Miranda y F.J. Botello-López. 2012. Registro notable de tres especies de mamíferos en la Reserva de la Biosfera Sierra Gorda de Guanajuato. Pp. 280-282, en: La Biodiversidad en Guanajuato: Estudio de Estado Volumen II (CONABIO, eds.). Comisión Nacional para el Conocimiento y Uso de la Biodiversidad (coNABIO)/ Instituto de Ecología del Estado de Guanajuato (IEE), Ciudad de México y Guanajuato. 
Charre-Medellín J.F., V. Sánchez-Cordero, G.E. Magaña-Cota, M. Álvarez-Jara y F.J. Botello. 2012a. Jaguarundi (Puma yagouaroundi) in Guanajuato, México. Southwestern Naturalist, 57:117-118.

Charre-Medellín J.F., V. Sánchez-Cordero, G.E. Magaña-Cota y F.J. Botello. 2012b. Estudio de la diversidad de mamíferos de talla mediana y grande del municipio de Victoria. Pp. 24-85, en: La Biodiversidad en Guanajuato: Estudio de Estado Volumen II (CONABIO, eds.). Comisión Nacional para el Conocimiento y Uso de la Biodiversidad (CONABIO)/Instituto de Ecología del Estado de Guanajuato (IEE), Ciudad de México y Guanajuato.

Charre-Medellín J.F., G. Magaña-Cota, T.C. Monterrubio-Rico, R. Tafolla-Muñoz, J.L. CharreLuna y F. Botello. 2016. Mamíferos medianos y grandes del municipio de Victoria, Reserva de la Biosfera Sierra Gorda Guanajuato, México. Acta Universitaria, 26(NE-2):62-70.

Chávez C., A. de La Torre, A. Bárcenas, R. Medellín, H. Zarza y G. Ceballos. 2013. Manual de fototrampeo para estudios de fauna silvestre. El jaguar en México como estudio de caso. Alianza WWF-Telcel, Universidad Nacional Autónoma de México. Ciudad de México, México.

CONANP (Comisión Nacional de Áreas Naturales Protegidas). 2005. Estudio previo justificativo para el establecimiento del área natural protegida Reserva de la Biosfera "Sierra Gorda de Guanajuato". Secretaria de Medio Ambiente y Recursos Naturales, México D.F.

conanp (Comisión Nacional de Áreas Naturales Protegidas). 2013. Programa de Manejo Reserva de la Biosfera Sierra Gorda de Guanajuato, México, documento para consulta pública, en cumplimiento a lo previsto por el artículo 65 de la ley general del equilibrio ecológico y la protección al ambiente general del equilibrio ecológico y la protección al ambiente [Internet]. Disponible desde: <http:/ simec.conanp.gob.mx/ficha.php?anp=109\&=11>. [Consultado el 1 de abril de 2017].

Elizalde-Arellano C., J.C. López-Vidal, E.Q. Uhart, J.I. Campos- Rodríguez y R. Hernández-Arciga. 2010. Nuevos registros y extensiones de distribución de mamíferos para Guanajuato, México. Acta Zoológica Mexicana (n. s), 26:73-98.

Iglesias-Hernández J.A., V. Sánchez-Cordero, G. Magaña-Cota, R. Bolaños, M. Aranda, R. Hernández-Arciga y F.J. Botello. 2008. Noteworthy records of margay, Leopardus wiedii and ocelot, Leopardus pardalis in the state of Guanajuato, Mexico. Mammalia, 72:347-349.

Iglesias-Hernández J.A., V. Sánchez-Cordero, G. Magaña-Cota, R. Bolaños y F.J. Botello. 2012. Diversidad de mamíferos medianos y grandes en el municipio de Xichú. Pp. 275-276, en: La Biodiversidad en Guanajuato: Estudio de Estado Volumen II (conABIO, eds.). Comisión Nacional para el Conocimiento y Uso de la Biodiversidad (CONABIO)/Instituto de Ecología del Estado de Guanajuato (IEE), Ciudad de México y Guanajuato.

Magaña-Cota G.E., J.F. Charre-Medellín, R. Hernández-Arciga, J. Iglesias, B. Chávez-Galván, R. Bolaños, R. Cecaira-Ricoy, V. Sánchez-Cordero y F. Botello. 2010. Primeros registros del murciélago vampiro de pata peluda (Diphylla ecaudata). Therya, 1:213-220.

Medellín R., D. Azuara, L. Maffei, H. Zarza, H. Bárcenas, E. Cruz, R. Legaria, I. Lira, G. Ramos-Fernández y S. Ávila. 2006. Censos y Monitoreo. Pp: 25-35, en: El Jaguar Mexicano en el Siglo XXI: Situación Actual y Manejo (Chávez C. y G. Ceballos, eds.). Comisión Nacional para el Conocimiento y Uso de la Biodiversidad - ALIANZA WWF TELCEL - Universidad Nacional Autónoma de México. Ciudad de México, México.

Mora-Villa L.A., C. Elizalde-Arellano y J.C. López-Vidal. 2014. Estudio de la comunidad de quirópteros de San Luis de la Paz, Guanajuato, México. Mastozoología Neotropical, 21:145-150. 
Ramírez-Albores J.E., A. Gordillo-Martínez y A.G. Navarro-Sigüenza. 2015. Registros notables y listado avifaunístico en un área de la Reserva de la Biosfera Sierra Gorda de Guanajuato, México. Revista Mexicana de Biodiversidad, 86:1058-1064.

Ramírez-Pulido J., N. González-Ruiz, A. L. Gardner y J. Arroyo Cabrales. 2014. List of recent land mammals of Mexico, 2014. Special Publications of the Museum of Texas Tech University, 63:1-69.

Sánchez O., G. Téllez-Girón y G. Magaña-Cota. 2009. Registros adicionales de murciélagos para Guanajuato. Acta Universitaria, 19:40-47.

Sánchez O., C. Elizalde-Arellano, J.C. López-Vidal, G. Magaña-Cota, G. Téllez-Girón, F. Botello y V. Sánchez-Cordero. 2012. Mamíferos silvestres. Pp. 263-274, en: La Biodiversidad en Guanajuato: Estudio de Estado (CONABIO, eds.). Comisión Nacional para el Conocimiento y Uso de la Biodiversidad (CONABIO)/Instituto de Ecología del Estado de Guanajuato (IEE), Ciudad de México y Guanajuato.

Sánchez O. G. Magaña-Cota, G. Téllez-Giron, W. López-Forment y G. Urbano-Vidales. 2014. Mamíferos no voladores de Guanajuato, México: revisión histórica y lista taxonómica actualizada. Acta Universitaria, 24:3-37.

Sánchez O., J.F. Charre-Medellín, G. Téllez-Girón, O. Báez-Montes y G. Magaña-Cota. 2016. Mamíferos silvestres de Guanajuato: actualización taxonómica y diagnóstico de conservación. Pp. 243-280, en: Riqueza y Conservación de los Mamíferos en México a Nivel Estatal (Briones-Salas, M., Y. Hortelano-Moncada, G. Magaña-Cota, G. Sánchez-Rojas y J.E. Sosa-Escalante, eds.). Instituto de Biología, Universidad Nacional Autónoma de México, Asociación Mexicana de Mastozoología A.C. y Universidad de Guanajuato, Ciudad de México.

SEmARnAt (Secretaría del Medio Ambiente y Recursos Naturales). 2010. Norma Oficial Mexicana NOM-059-SEMARNAT-2010, Protección ambiental-Especies nativas de México de flora y fauna silvestre-categorías de riesgo y especificaciones para su inclusión, exclusión o cambio-Lista de especies en riesgo. Diario Oficial de la Federación, 30 de diciembre de 2010, 78 p.

Semarnat (Secretaría de Medio Ambiente y Recursos Naturales). 2007. Decreto por el que se declara área natural protegida con el carácter de reserva de la biosfera la zona conocida como Sierra Gorda de Guanajuato localizada en los municipios de Atarjea, San Luis de la Paz, Santa Catarina, Victoria y Xichú en el estado de Guanajuato. Diario Oficial de la Federación, 2 de febrero de 2007, primera Sección. México, D.F. 